\title{
Germanica
}

\section{Das Schreiben unter digitalen Bedingungen}

Hannes Bajohr im Interview mit Robin-M. Aust

\section{Robin-M. Aust}

\section{CpenEdition}

\section{Journals}

Édition électronique

URL : http://journals.openedition.org/germanica/7191

DOI : 10.4000/germanica.7191

ISSN : 2107-0784

Éditeur

Université de Lille

\section{Édition imprimée}

Date de publication : 26 juin 2019

Pagination : 201-212

ISBN : 978-2-913857-42-1

ISSN : 0984-2632

\section{Référence électronique}

Robin-M. Aust, „Das Schreiben unter digitalen Bedingungen“, Germanica [Online], 64 | 2e trimestre 2019, Online erschienen am: 01 Januar 2021, abgerufen am 24 Februar 2021. URL: http:// journals.openedition.org/germanica/7191 ; DOl: https://doi.org/10.4000/germanica.7191 
Entretien 



\section{Das Schreiben unter digitalen Bedingungen}

Hannes Bajohr im Interview mit Robin-M. Aust

Anfang 2018 bei Suhrkamp veröffentlicht, versammelt Hannes Bajohrs Lyrikband Halbzeug eine Vielzahl von Gedichten unterschiedlichster, digitaler Genese: ein Hochgeschwindigkeitsrauschen aneinandergereihter Karriereratgeberfloskeln stehen neben von Spracherkennungsprogrammen missverstandenen Texten; Kafka und die Gebrüder Grimm stehen neben Versatzstücken aus Klimaschutzberichten. Alle Gedichte in Halbzeug basieren auf bestehenden, oft auch kanonischen Texten, die größtenteils autonom mithilfe digitaler Prozesse und unterschiedlicher, teils zweckentfremdeter Software transformiert worden sind. Ursprungsmaterial, Konzept, Prozess und lyrisches Endergebnis gehen somit einerseits Hand in Hand, stehen andererseits in einem ambivalenten Spannungsverhältnis zueinander und formen so erst zusammen die Botschaft der Texte. Halbzeug setzt damit unterschiedliche Tendenzen der Nachkriegs- und Gegenwartslyrik fort: so ist die konzeptuelle Nähe zu readymade-Lyrik z.B. eines Gerhard Rühm, Oskar Pastior oder den Literaturexperimenten von Oulipo schnell gezogen. Wenn Bajohr dagegen kanonische Gedichte durch die Synonymsuche von Microsoft Word verfremden lässt, so lässt sich dies durchaus als fortgesetzte, diesmal vom Wortfindungsprozess des Künstlers losgelöste Hermetisierung poetischer Sprache verstehen.

Die Gedichte stellen so nicht nur die Frage nach der Funktion des Digitalen im Alltag und im Kunstbetrieb und präsentieren neue Möglichkeiten der Texttransformation, die das poetische Arsenal des 
Lyrikers - gewissermaßen im Geiste des ,open source - erweitern: Bajohr gibt die verwendete Software und das Konzept der Transformation preis und gibt dem Leser so selbst konkrete Werkzeuge zur Transformation an die Hand, sodass jener eingeladen wird, auf dieser Basis selbst ähnliche Transforme zu erstellen - der Autor gibt seine Vormachtstellung über das Werk somit auch performativ auf und stellt den digitalen Prozess und das Konzept in den Vordergrund. Hannes Bajohr, 1984 in Berlin geboren, promovierte an der Columbia University zu Hans Blumenbergs Sprachtheorie, ist Übersetzer Judith N. Shklars und Kenneth Goldsmiths und Mitherausgeber von Peter Weiss' Briefen. Er publizierte zum Werk Hannah Arendts und Judith N. Shklars sowie zur Literatur- und Politiktheorie, aber auch zum Verhältnis von Digitalität und Literatur ${ }^{1}$. 2016 gab er den Sammelband Code und Konzept. Literatur und das Digitale heraus ${ }^{2}$. Als Schriftsteller betätigt sich Bajohr ebenfalls auf dem Gebiet digitaler Literatur: 2015 veröffentlichte er den Roman Durchschnitt ${ }^{3}$, der auf den Texten aus Marcel Reich-Ranickis RomanKanon basiert; ebenfalls 2015 erschien Timidities $^{4}$, das unter anderem Textfetzen aus einem BDSM-Forum und einem Sexratgeber verwendet; 2017 folgte Monologue ${ }^{5}$, das Passagen aus Timidities erweitert und dem Leserbriefe an eine queere Beziehungskolumne zugrunde liegen. Zuletzt kam 2018 der Lyrikband Halbzeug. Textverarbeitung im Suhrkamp Verlag heraus. Mit Gregor Weichbrodt betreibt Bajohr das Textkollektiv 0x0a ${ }^{6}$. Derzeit ist er wissenschaftlicher Mitarbeiter am Leibniz-Zentrum für Literatur- und Kulturforschung in Berlin.

Robin-M. Aust: Halbzeug ist nicht Ihre erste größere literarische Veröffentlichung, die auf der Transformation bestehender Texte durch digitale Techniken basiert. Bereits Monologue und Durchschnitt entstanden durch ähnliche, auf digitalen Techniken fußende Konzepte. Wie kam Ihnen die Idee zu diesem Projekt?

Hannes Bajohr: Was Durchschnitt betrifft, hatte ich mich zu diesem Zeitpunkt schon länger - vor allem im Kontext des Textkollektivs 0x0a, das ich mit Gregor Weichbrodt betreibe - mit der literarischen Verarbeitung größerer Textkorpora beschäftigt. Ein grundsätzliches Problem bei dieser Arbeit ist die Frage, wie man das Korpus abgrenzt - wo man gewissermaßen zu sammeln aufhört. Es geht also um den Umgang

1. — Ein vollständiges Schriftenverzeichnis findet sich online unter http://www. zfl-berlin.org/person/bajohr.html (Zugriff am 02.05.2019).

2. - Hannes Bajohr (Hrsg.), Code und Konzept. Literatur und das Digitale, Berlin, Frohmann, 2016.

3. - Hannes Bajohr, Durchschnitt, Berlin, Frohmann/0x0a, 2015.

4. - Hannes Bajohr, Timidities, Berlin, Readux, 2015.

5. - Hannes Bajohr, Monologue, Berlin, Frohmann/0x0a, 2017.

6. — https://0x0a.li (Zugriff am 02.05.2019). 
mit Kontingenz und Komplexität. Der Roman-Kanon von Reich-Ranicki reduziert beides, und zwar doppelt. Einerseits gibt er per fiat vor, wo das Textmaterial beginnt und aufhört; und zweitens ist der Kanon selbst ein Mittel, mit der Masse des Unlesbaren umzugehen, indem sie aufs vermeintlich Wesentliche eingedampft wird. Durchschnitt denkt diesen Gedanken lediglich fort, nur, dass es das Kritiker-Kriterium - literarische Qualität, vielleicht auch literaturgeschichtliche Repräsentativität - durch ein rein quantitatives ersetzt: Die durchschnittliche Satzlänge, die bei diesem Korpus 18 Wörtern entspricht. Durchschnitt enthält alle Sätze dieser Länge, alphabetisch sortiert und nach Buchstaben in Kapitel sortiert.

Bei Monologue, das als Quellmaterial eine Sex- und Beziehungskolumne verwendet, stand eine soziologische Beobachtung am Anfang: Die Autor*innen der eingesandten Fragen neigen dazu, sich zu identifizieren, etwa „I am a 24-year old white bisexual male.“ Nur das Identifikatorische aus diesem Korpus herauszusuchen, also alle Sätze, die mit „I'm“ oder „I am“ beginnen, und sie dann mit zufälligen Konjunktionen zu verknüpfen, lag da fast auf der Hand.

[Beispiel 1: Was man muss (Managementkorpus), Auszug]

Sie müssen tough sein.

Sie müssen ehrlich sein.

Sie müssen es nur wollen.

Sie müssen emotional sein.

Sie müssen sich entscheiden!

$[\ldots]$

Sie müssen es tun ob Sie wollen oder nicht.

Sie müssen die Fragesteller konsequent führen.

Sie müssen Ihre Zuhörer zusätzlich motivieren.

Sie müssen sich nicht für eine Absage entschuldigen.

Sie müssen vor Ihrem Vortrag immer auf die Toilette?

$[\ldots]$

Sie müssen fürchten, dass Ihre Mittelmäßigkeit irgendwann durch

einen Besseren offenbar wird.

Sie müssen wissen, was von Ihnen erwartet wird und wie Sie diese Erwartungen erfüllen können.

Sie müssen Ihre Mindset-Eigenschaften nutzen, um vom Netzwerker zum Supervernetzer zu werden.

Sie müssen sich von dem Stapel der anderen Lebensläufe abheben - oder das Spiel ist gelaufen.

Sie müssen nicht auf guten Stil, Grammatik, Zeichensetzung, Satzbau und vollständige Sätze achten.

$[\ldots]$ 
Sie müssen Ihr Leben, alles, was Sie bisher getan haben, Ihrekompletten Pläne und Ziele ändern - oder Sie werden sterben?

RMA: Im Falle der Texte in Halbzeug passen Mechanismus der Textgenese und Inhalt häufig gut zueinander. Was man muss (Managementkorpus) (Beispiel 1) basiert auf der Länge nach geordneten Auszügen aus Erfolgsratgebern, die mit ,Sie müssen" beginnen und sich so in ein Crescendo der Handlungsanweisungen steigert. Was ist zuerst da - die Idee, auf die Sie einen Prozess hin konzipieren und die thematische Richtung, in die das Transform gehen soll, oder der Prozess, der dann ein interpretierbares Ergebnis liefert?

HB: Konzeptidee und Umsetzung stehen in einer Art Feedback-Loop, und auch das wieder doppelt: Einerseits ist es oft das Material, das die Idee überhaupt erst vorgibt - in diesem Fall hat es der imperative Ton der Ratgeberbücher nahegelegt. Es gibt also nicht die absolute Autarkie des konzeptuellen Einfalls. Andererseits kann ich die Effektivität und Prägnanz eines Konzepts immer erst am Endprodukt messen. Das straft den Anspruch einer rein konzeptuellen Literatur (die ich auch gar nicht vertrete) Lügen, denn eigentlich sollte die Ausführung ja einerlei sein, wenn nur das Konzept stimmt. So ist es aber nicht; vielmehr kann man die Plausibilität des Konzepts erst an seinem „Output“ ersehen, sich vorher auch gar nicht vorstellen.

$\boldsymbol{R M A}$ : Sie beziehen sich explizit auf Nietzsches oft zitierte Sentenz „, Unser Schreibzeug arbeitet mit an unseren Gedanken "-aber wo sehen Sie die ,Bedeutung', die ,Botschaft' - im Schreibzeug, dem Code? Im Text? In der Differenz zwischen Original und Transformen? Im Konzept?

HB: Nietzsche war gewissermaßen der Aufhänger für die Auseinandersetzung mit dem Einfluss des Schreibgeräts auf die literarische Produktion. Und heute ist das Schreibgerät ja so gut wie ausschließlich digital. Wieso wird das so selten literarisches Thema? Oder wenn, warum nur, indem man in einem Roman mal eine E-Mail schreibt oder einen Facebook-Chat fingiert? Mich interessiert also die Bedeutung, die sich in der Reflektion auf das Schreiben unter digitalen Bedingungen findet. Dazu gehören dann im Weiteren auch Fragen wie Urheberschaft, Transkodierung, Konzeptualität und so weiter.

Übrigens steht Nietzsche - dessen Malling-Hansen-Schreibkugel ich auf der Ebene der Binärdatei verfremdet habe - ja nur am Anfang; die letzte Bearbeitung dieses Zyklus, der Schreibzeug heißt, ist selbst

7. - Hannes Bajohr, „Was man muss“ (Managementkorpus), in: Ders., Halbzeug. Textverarbeitung, Frankfurt a.M., Suhrkamp, 2018, S. 12-14. 
nur noch sekundäres Schreibzeug: ein FORTRAN-Skript von Alison Knowles und James Tenney.

$\boldsymbol{R M A}$ : Sehen Sie eine werkübergreifende Botschaft in Ihren Texten oder unterscheidet sich das von Text zu Text, von Konzept zu Konzept?

HB: Das ist sehr unterschiedlich. Aber wenn es eine weitere „Botschaft" gibt, dann ist das vielleicht gerade dieses Plädoyer gegen digitalen Inhaltismus: Digitalität kann Gegenstand von Literatur sein, ohne auf der semantischen Ebene vorzukommen, und kann sich stattdessen in den Produktionsbedingungen abspielen, die bei der Lektüre einbezogen werden können, aber nicht müssen. Viele der Texte in Halbzeug funktionieren ja auf zwei Ebenen - einmal als digital hergestellte Gedichte, die etwas über textliche Verfüg- und Bearbeitbarkeit sagen, aber dann eben auch als digital hergestellte Gedichte, die man auch unabhängig von ihrem Herstellungsprozess lesen kann.

$\boldsymbol{R M A}$ : Ihre Texte haben oft eine auffällige Kohärenz, die zum Teil vielleicht auch erst im Rezeptionsprozess des Lesers entsteht - greifen Sie in den generierten Text nachträglich ein, rearrangieren oder korrigieren Sie beispielsweise Inhalt oder Grammatik?

HB: Das ist von Text zu Text unterschiedlich und hängt von der angewandten Technik ab. Am einen Ende des Spektrums stehen die Texte aus dem Zyklus Maschinensprache - zum Beispiel die auf ein visuelles Gedicht von Apollinaire angewandte Texterkennung, die nur Buchstabensalat ausgibt -, in die ich gar nicht eingegriffen habe. Die einzige „Intervention“" besteht in der Entscheidung zur Veröffentlichung. Am anderen Ende des Spektrums stehen dann die Texte aus Automatengedichte, bei denen ich mittels Zufallsoperationen vorhandene Texte neu mische, dann aber sehr frei, und das heißt subjektiv, arrangiere und reduziere. Grammatische Korrekturen finden schließlich in den Texten statt, für die ich kanonische Gedichte Wort für Wort mit der Synonymfunktion aus Microsoft Word umformuliere (Beispiel 2). Allen Texten ist aber gemein, dass sie immer auf bereits vorhandenem Text als Ausgangsmaterial beruhen und ich ihnen nie etwas hinzufüge, was nicht bereits materialiter in ihnen steckt.

[Beispiel 2: Schreibkram]

Wenn der Nachrichtendienst im Dunkel vorrückte und der Trabant schupste die Ausfälle unter den Ausstieg:

Sie entstünden wie Cherubim

in ihren getünchten Formen 
und ankerten gelinde in Busch und Feld ${ }^{8}$.

RMA: In einem Artikel für die NZZ schreiben Sie, der Autor sei ,, kein Originalgenie mehr und wird Kurator des schon Existierenden; auch der Schöpfungsprozess verschiebt sich auf eine sekundäre Ebene: Es wird geschrieben, um dann schreiben zu lassen, denn es ist der vom Autor konstruierte Code, nicht mehr der Autor unmittelbar selbst, der den Text hervorbringt. "9 - und wem gehört der fertig generierte Text? Goethe, Bajohr, dem Computer? Ist es dennoch ,Ihr' Text?

HB: Die Autorfunktion selbst kann man schwer abschaffen. Es steht immer noch mein Name auf dem Buch, genau, wie es meine Entscheidung war, diese und nicht andere Texte auszuwählen. Auch ist der Schreibprozess immer noch einer, der aus einer - mal längeren, mal kürzeren - Reihe von Entscheidungen besteht. Nur finden diese Entscheidungen auf einer anderen, operationellen, eben sekundären Ebene statt, bestehen in der Formulierung von Regeln statt in der Formulierung von Sätzen; sie sind damit auch transparenter und einfacher zu befragen. Aber wichtig ist für mich, sich des Unterschieds bewusst zu sein, der zwischen Fantasien vom Tod des Autors mit maschineller Hilfe besteht (woran ich nicht glaube) und der Abschaffung des Autorgenies als Authentizitätsquelle (was mir sympathisch ist). Literaturwissenschaftlich ist das natürlich ein alter Hut, aber ich bitte Sie, sich einmal die Selbstpräsentation und nicht zuletzt die Berichterstattung über Bücher und Autor*innen und das Verhältnis von Vita zu Werk anzusehen - da ist noch ziemlich viel Genie abzutragen, auch in der Literaturkritik.

$\boldsymbol{R M A}:$,Everyone is an artist “- aber treffen Werke wie Ihres auch eine Aussage über das Urheberrecht nichtmenschlicher Urheber? Man denke hier auch - etwas polemisch - an die vor einiger Zeit geführte Diskussion um die von einem Affen gemachten Selfies und die Frage, ob die Fotos nun dem Besitzer der Kamera oder dem Affen als Fotografen gehören.

HB: Die Frage ist interessant, aber im Fall des Affen doch etwas anders gelagert als wenn es um Skripte geht, schließlich ist das Programm ja selbst Produkt eines Schöpfungsprozess durch die Programmierenden. Aber natürlich findet bei der Arbeit mit allem Gerät eine Form von

8. - Hannes Bajohr, „Schreibkram“, ebd., S. 92. „Schreibkram“ basiert auf dem Gedicht „Briefwechsel“ von Ilse Aichinger (1978): „Wenn die Post nachts käme / und der Mond / schöbe die Kränkungen / unter die Tür: / Sie erschienen wie Engel / in ihren weissen Gewändern / und stünden still im Flur.“ (Ilse Aichinger, „Briefwechsel“, in: Dies., Verschenkter Rat. Gedichte, hrsg. v. Richard Reichensperger, Frankfurt a.M., Fischer, 1991, S. 22).

9. - Hannes Bajohr, „Unermüdlich dichtet das Maschinchen“, Neue Zürcher Zeitung (27.02.2018), online verfügbar unter: https://www.nzz.ch/feuilleton/unermuedlich-dichtet-das-maschinchen-ld.1351544 (Zugriff am 02.05.2019). 
Selbstentäußerung statt, bei der zu fragen wäre, wie weit man sie treiben kann. Ich halte da die Idee des extended mind von Andy Clark und David Chalmers für sehr plausibel: Sobald „Geist" nicht mehr nur in meinem Kopf stattzufinden braucht, sondern in der Interaktion zwischen verkörpertem Selbst und seiner Umgebung - mit Stift und Notizen nicht weniger als mit Tastatur und Bildschirm - muss man Urheberschaft ohnehin eher als Konglomerat verstehen, das sich nicht ohne Weiteres lokalisieren lässt.

RMA: Ist der hinter dem Transform stehende Code lediglich Mittel zum Zweck, bloßes ,Schreibzeug 'oder selbst Kunst, Poesie?

HB: Ich gehe von einer Analogie von Code und Konzept aus: Was den Text hervorbringt, ist das Konzept, das programmatisch, also per Code, umgesetzt werden kann, aber nicht muss. Insofern lege ich weniger Wert auf die exakte Umsetzung des Konzepts auf Codeebene als es die Bewegung der Code Poetry tut, die den Quelltext ganz streng als Teil des Werkes versteht, oder in ihrer Literatur die Ästhetik des Codes verwendet. Auch veröffentliche ich die Skripte nicht, die ich verwende - mir reicht es, wenn ihre generelle Machart über das Verständnis des Konzepts erfasst werden kann, das dann so oder auch anders zu programmieren wäre. Und schließlich verwende ich oft proprietäre Software, arbeite also vielfach gar nicht auf Codeebene.

\section{[Beispiel 3: Töten (Korpusfabel III)]}

[...] Da zog er sein Schwert und drohte sie zu töten, wo sie ihm nicht gehorchte und zwang sie damit, daß sie es versprach. Daheim wußte er nicht, an wem er seinen Zorn und Ärger auslassen sollte, bis ihm böse Gedanken kamen und er beschloß, seinen Bruder zu töten. Den jüngsten tötete Hermenfried auf Anstiften seiner Gemahlin Amalaberga, einer Tochter Theodorichs von Franken. Der Knecht nahm sie in seinen Mantel, ritt in den Wald und wollte sie töten. Der König hieß in jähem Zorn den Grafen töten. Der König ließ seinen Rat versammeln und beschloß, den dritten Teil des Volkes töten zu lassen. Der Mann geriet in Schulden und ward ganz arm, so daß er in Verzweiflung seine Frau mit einem Messer tötete, dann sich selbst eine Kugel durch den Kopf schoß. Die Hunnen verheerten die ganze Stadt; was von Männern darin war, töteten sie durchs Schwert, um die Weiber und Kinder aber losten sie. [.... ${ }^{10}$.

10. — Hannes Bajohr, „Töten“ (Korpusfabel III), in: Ders., Halbzeug..., a.a.O., S. 26-27. Als Textgrundlage dienen alle Sätze aus den Grimm'schen Kinder- und Hausmärchen, die das Lemma ,töten` enthalten. 
RMA: Welche praktischen Konsequenzen haben das Urheberrecht der von Ihnen verwendeten Texte und die Quellenlage auf Ihre Arbeit? Für Ihre Werke benutzen Sie unterschiedliche Quellentexte, von Gedichten und Grimms Märchen (Beispiel 3) über den Deutschen Referenzkorpus (DeReKo) bis hin zu Aufgefundenem wie beispielsweise den erwähnten Leserbriefen an eine Sexkolumne - vor allem also Texte, die frei verfügbar, maschinentextlich lesbar oder für einen Menschen zumindest abtippbar sind.

HB: Rein urheberrechtlich habe ich keine Probleme, weil ich immer Transformationen vornehme und so aus dem Ausgangsmaterial ein sichtbar ,eigenständiges Werk" mache (wie ja der juristische Terminus lautet). Das ist bei anderen Spielarten der konzeptuellen Literatur anders, vor allem im Bereich der Appropriationsliteratur, wo also vorhandene Texte eins zu eins übernommen werden - dort ist mir bisher aber nur ein Fall bekannt, bei dem es zu rechtlichen Konsequenzen gekommen ist. Eine andere Sache ist natürlich die Quellenlage: Voraussetzung für digitale Verarbeitung ist die Verfügbarkeit digitalen Texts. Das hat zur Folge, dass ich vornehmlich digitalen Text aufbereite und sehr viel seltener analogen Text digitalisiere. Ich scrape etwa Datingprofile, die ja bereits digital vorhanden sind, nur noch nicht in einer durchsuchbaren Form, aber nur ausnahmsweise scanne ich analogen Text und digitalisiere ihn, wie im Fall der kanonischen Gedichte, von denen nicht alle schon digital verfügbar waren. Mein Material unterliegt in dieser Hinsicht sicher einer gewissen Befangenheit, was man vielleicht availability bias nennen könnte. Inzwischen liegt allerdings so viel historischer Text digitalisiert vor, dass ich mich frage, ob man hier wirklich eine signifikante Verzerrung feststellen könnte.

RMA: Sie geben am Ende der einzelnen Texte in Halbzeug an, welche Quellen und Tools Sie benutzt haben - laden Sie den Leser ein, es Ihnen nachzutun und selbst per Code und Software zum Autor transformierter Literatur und Schöpfer transformierender Prozesse und Konzepte zu werden?

HB: Ja. Das Buch ist ja gewissermaßen eine Werbebroschüre für die digitale Literatur. Die Schnittmenge zwischen denen, die mit digitaler Technik umgehen können, und denen, die literarisch produzieren, ist noch sehr klein. Das ändert sich langsam. Indem ich die Tools nenne, möchte ich zumindest den ersten Schritt hin zu anderen Experimenten damit leichter machen.

\section{RMA: Ist (Ihre) Literatur also Open Source?}

HB: Ich glaube an so etwas wie literarische Transparenz - ich möchte, dass man versteht, wie der Text gemacht wurde, auch wenn andere mit denselben Mitteln wahrscheinlich anderes produzieren würden. Das 
Pendant zu Open-Source-Literatur wäre übrigens proprietäre Literatur, was mir als Bezeichnung für Genieästhetik und das Verständnis von Autor*innen als Black Boxes ganz gut gefällt.

$\boldsymbol{R M A}$ : Sehen Sie sich in einer bestimmten literarischen Tradition? Ähnliche Transformationsmechanismen, wenn auch nicht algorithmusbasiert, finden sich ja beispielsweise auch bei Oulipo.

HB: Die ganze experimentelle literarische Tradition ist zumindest ein gutes Argument gegen Einwände, die digitaler Literatur ihre literarische Qualität absprechen wollen. Die müssen sich dann den Vorwurf gefallen lassen, literaturhistorisch vergesslich zu sein.

$\boldsymbol{R M A}$ : Konzepte wie ,glitch art" und ,, data moshing “ sind inzwischen Teil einer popkulturellen Ästhetik geworden, zumeist bleibt dies aber noch auf einer oberflächlichen, rein ästhetischen Ebene - in Ihren Texten und Transformen fallen Genese, Form und Inhalt stärker zusammen. Lassen sich ähnliche Konzepte Ihrer Meinung nach auch auf andere Medien und Kunstformen wie Musik oder Film übertragen?

HB: Das weiß ich nicht - aber Literatur hat den Vorteil, dass ihr Medium auch das Medium ihrer Kritik und Erklärung ist. Vielleicht macht es das für andere Kunstformen schwerer. Aber gut möglich, dass das nur ein Vorurteil von Literaturschaffenden ist.

$\boldsymbol{R M A}$ : Halbzeug erscheint zu einem Zeitpunkt, an dem das Digitale in Form sozialer Medien langsam vom Raum der grenzenlosen Möglichkeiten zu einer gefährlichen Echokammer wird. Dieser entgrenzte Raum bringt gleichzeitig aber immer neue Formen und Gattungen künstlerischen Ausdrucks hervor, während an anderer Stelle nach Einhaltung der Urheberrechte, Zensur und Regulierung verlangt wird. Wie setzt sich diese Entwicklung Ihrer Meinung nach fort?

HB: Das weiß ich nicht. Es kann sein, dass die Anpassung der Rechtslage Einiges an ästhetischem Potenzial vernichtet. Andererseits wird die Masse an potentiellem Material zur literarischen Verwertung immer größer. Also abwarten - „die Zukunft ist noch nicht passiert“11.

$\boldsymbol{R M A}$ : Das Internet ist voller kreativer, mal simpler, mal komplexer Bots und Textgeneratoren, oft auch mit subversiver Ausrichtung. Ist ein Bot, der auf Mausklick aus Trump-Tweets neue Markovketten generiert, lediglich eine amüsante, zynische Spielerei, oder tatsächlich ein Werkzeug sozialen, politischen Wandels?

11. - So der Titel eines Gesprächs von Hannes Bajohr mit Holly Melgard, online verfügbar unter: https://www.kunstforum.de/artikel/holly-melgard/ (Zugriff am 02.05.2019). 
HB: Das kommt darauf an, ob man Literatur überhaupt politisches Wirkungspotenzial unterstellt. Zumindest ist die Anverwandlung und Umwandlung der Worte politisch Mächtiger kein harmloser Akt.

RMA: Können Sie weitere Projekte oder Werke, deren Genese auf digitalen Techniken basiert, empfehlen?

HB: Zuerst natürlich Gregor Weichbrodt; mit dem ich 0x0a betreibe und der noch viel mehr als ich generativ arbeitet; sein Buch I Don't Know ${ }^{12}$ etwa basiert auf der gesamten Wikipedia. Er macht auch Twitter-Bots, wie den@bot_on_holiday, der Google-Street-View und Bilderkennung kombiniert und so eine unendliche Reiseerzählung produziert. In den USA bauen Ranjit Bhatnagar und Allison Parrish ebenfalls Bots und haben, wie Nick Montfort, interessante Projekte zum National Novel Generation Month ${ }^{13}$ eingereicht. Im deutschsprachigen Raum wäre vor allem Jörg Piringer zu nennen, der schon seit den Neunzigerjahren dabei ist und gerade das Buch Datenpoesie ${ }^{14}$ herausgebracht hat. Andreas Bülhoff überträgt mit Sync ${ }^{15}$ eine serielle Publikationsweise aus der ZineKultur ins Digitale. Und Jasmin Meerhoff macht unter nervousdata.com digitalen Cut-Up in der Tradition von Burroughs und Gysin mittels der Kommandozeilen-Texterkennung tesseract.

Ich hoffe auch, dass sich hier auf einer sekundären Ebene noch mehr tut - dass man nicht gleich „Digitalautor*in“" werden muss, um mit digitalen Techniken zu arbeiten, sondern auch mal ein Kapitel in einem andernfalls eher klassischen Roman generieren lässt, oder sich zur Kopfentlüftung mal am Automatengedichtautomaten ${ }^{16}$ versucht.

\section{RMA: Welche zukünftigen Projekte stehen in nächster Zeit an?}

HB: Ich probiere im Augenblick die Möglichkeiten von Machine Learning für die Literaturproduktion aus. Das heißt konkret, dass ich ein Programm Datensätze lernen und auf deren Grundlage dann ähnliche ausgeben lasse. Ein erstes Ergebnis erschien letzten Oktober in der Zeitschrift tau ${ }^{17}$. Für „Dreiunddreißig neue Sprichwörter" habe ich etwa die Liste deutscher Sprichwörter aus der Wikipedia als Lerndatensatz verwendet und neue generiert („Wo kein Sau, mild ihr einen Boden“, „Ein Schwere sind der Tod ist den Mann auf dem Augen“, „Wenn der Bauer'

12. - Gregor Weichbrodt, I don't know, Berlin, Frohmann/0x0a, 2016.

13. — https://nanogenmo.github.io/ (Zugriff am 02.05.2019).

14. - Jörg Piringer, Datenpoesie, Klagenfurt, Ritter, 2018.

15. — http://sync.abue.io/ (Zugriff am 02.05.2019).

16. - http://www.hannesbajohr.de/automatengedichtautomat/index.php (Zugriff am 02.05.2019).

17. - Vgl. Hannes Bajohr, „Lernprozesse“, tau. zeitschrift für literatur, 02/2018, S. 9-14. 
hilt glüt, aber weker dem andern Brot ein Gebier“, etc.). Interessant ist hier für mich zu sehen, welche Regelhaftigkeit das Programm im Input erkennt (,wo“ oder „wenn“ als erstes Wort, der Bauer als Protagonist, der Apostroph). Die Regel selbst kann ich nur erschließen, gebe also selbst nur noch die Anweisung, eine Regel zu generieren, nach der generiert wird. Hier könnte man eigentlich von tertiärer Autorschaft sprechen, denn wo dem klassischen Selbstschreiben das Schreibenlassen durch Algorithmen und Konzepte gegenübersteht, ist das noch eine Stufe weiter entfernt: Statt die Regel zu entwerfen, nach der gedichtet wird, entwerfe ich die Regel für diese Regel.

Das Interview wurde im Frühjahr 2019 per Mail geführt. Vielen Dank an Hannes Bajohr für die bereitwillige Beantwortung der Fragen. 
